J. Elsner ${ }^{1,2}$
U. Forssmann

\section{Chemokinrezeptor-Antagonisten bei atopischen Erkrankungen}

\author{
Chemokine Receptor Antagonists in Allergic Diseases
}

\section{Zusammenfassung}

Die Inzidenz allergischer Erkrankungen nimmt in den westlichen Industrieländern stetig zu. Hieraus resultieren für die Therapie sehr hohe Kosten (USA jährlich ca. 18 Milliarden Dollar). Aus diesem Grund ist die Forschung darauf ausgerichtet, neue Medikamente zur Therapie allergischer Erkrankungen zu finden. In diesem Übersichtsartikel stellen wir einen neuen Therapieansatz vor: Die Chemokinrezeptor-Antagonisten bei atopischen Erkrankungen.

\section{Abstract}

The incidence of allergic diseases increases in the western industrial countries costing the health care system $\$ 18$ billion (USA) annually. Therefore, institutional and industrial research focus on new concepts to find therapies preventing allergies or stopping the symptoms. Herein, we postulate a new concept in the treatment of allergies: Chemokine receptor antagonists for atopic diseases.

\section{Einleitung}

Chemokine sind chemotaktische Peptide, denen durch die Rekrutierung von Leukozyten eine zentrale Funktion bei immunologischen und entzündlichen Prozessen zukommt. Neben ihrer Eigenschaft, Entzündungszellen in das Entzündungsgebiet anzulocken (Chemotaxis), vermitteln Chemokine am Ort der Entzündung durch die Freisetzung toxischer Metabolite aus Granulozyten (reaktive Sauerstoffspezies) eine Gewebsschädigung [1].

Chemokine werden in Abhängigkeit ihrer konservierten Zysteine im N-terminalen Ende in 4 Familien unterteilt: CXC-Chemokine (CXCL), CC-Chemokine (CCL), C-Chemokine (XCL) und CXXXCChemokine (CX3CL). Sie binden an Chemokinrezeptoren, die zur Gruppe der Gi-Protein-gekoppelten 7-transmembranösen Rezeptoren gehören: Ursprünglich war es allgemein anerkannt, dass sich die jeweiligen Unterfamilien in ihrer biologischen Aktivität durch Stimulation verschiedener Leukozyten unterscheiden. CXC-Chemokine vermittelten durch Aktivierung neutrophi- ler Granulozyten die akute Entzündungsreaktion, während CCChemokine durch die Einwanderung eosinophiler und basophiler Granulozyten, Monozyten oder Lymphozyten die chronische Entzündung regulieren. Neuere Untersuchungen konnten jedoch zeigen, dass diese Regel zahlreiche Ausnahmen hat und die Wirkung der Chemokine wesentlich komplexer ist [2].

Aufgrund ihrer zentralen Bedeutung für die Rekrutierung und Aktivierung von Entzündungszellen, sind Chemokine und ihre Rezeptoren in den Fokus für neue Therapieformen gerückt. Hierbei stehen vor allem Substanzen im Mittelpunkt, die an den Chemokinrezeptor binden und die Wirkung des Chemokins damit antagonisieren. In dieser Übersicht beschreiben wir die Rolle von Chemokinrezeptor-Antagonisten als mögliche neue Therapiestrategie bei atopischen Erkrankungen. 
Der CC-Chemokinrezeptor CCR3 spielt eine herausragende Rolle für allergische Erkrankungen. Dieser Rezeptor bindet Eotaxin und seine Verwandten Eotaxin-2 und Eotaxin-3. Darüber hinaus bindet CCR3 die natürlichen Chemokine RANTES, MCP-2, MCP-3, MCP-4, MEC sowie HCC-1(9-74) > 9 [1]. Interessanterweise sind die CXC-Chemokine Mig, IP10 und I-TAC, die den CXC-Chemokinrezeptor 3 und damit T-Lymphozyten aktivieren, potente Antagonisten am CCR3 [2].

Seine besondere Bedeutung für die allergische Entzündung hat der CCR3 aufgrund seiner selektiven Expression auf eosinophilen [3] und basophilen [4] Granulozyten, Mastzellen [5] sowie auf Th2-Zellen [6]. Diese Zellen exprimieren unter definierten Bedingungen den CCR3 und lassen sich durch das Th2-Chemokin Eotaxin chemotaktisch aktivieren. In einer Studie mit einer CCR3-Knock-out-Maus konnte gezeigt werden, dass der CCR3 sowohl für die Einwanderung eosinophiler Granulozyten in das Lungengewebe als auch für die bronchiale Hyperreagibilität mitverantwortlich ist, wobei letztere Beobachtung nur dann zu beobachten war, wenn die Tiere über einen epikutanen Weg sensibilisiert worden sind $[7,8]$. In einem Mausmodell für die atopische Dermatitis konnte ebenfalls gezeigt werden, dass der CCR3 für die Einwanderung eosinophiler Granulozyten essenziell ist [8]. Aus diesem Grund wird dem CCR3 eine besondere Bedeutung bei der allergischen Entzündung zugerechnet, und er ist deshalb ein potenzielles Ziel für die Generierung von Chemokinrezeptor-Antagonisten $[9,10]$. Hierzu zählen Peptid-Antagonisten, die sich von Chemokinen ableiten bzw. klein-molekülige Substanzen, auf die wir im nächsten Abschnitt eingehen werden.

Chemokinrezeptor-Antagonisten als zukünftige Therapie bei allergischen Erkrankungen?

\section{Peptide als Chemokinrezeptor-Antagonisten}

Die ersten klassischen Chemokinrezeptor-Antagonisten wurden durch Modifikation im N-terminalen Ende hergestellt. Diese Region des Chemokins ist nämlich für die Bindung am Rezeptor und Entfaltung der biologischen Aktivität entscheidend [11 - 14]. So sind verschiedene Chemokinrezeptor-Antagonisten wie Met-RANTES, AOP-RANTES, MCP-1(2-76), RANTES(3-68), MCP-2(6-76) durch An- oder Ablagerung von Aminosäuren bzw. chemische Modifikation im N-terminalen Ende der Chemokine konstruiert worden $[12,13,15-20]$.

Interessanterweise existieren aber auch einige Chemokinrezeptor-Antagonisten physiologisch in vivo. Manche Chemokine können zudem potenziell in vivo von bestimmten Peptidasen zu Chemokinrezeptor-Antagonisten umgewandelt werden. Ein gutes Beispiel hierfür ist die Dipeptidylpeptidase IV, die als Oberflächenantigen (CD26) beispielsweise auf Lymphozyten exprimiert wird. Darüber hinaus findet sich dieses Enzym auch im Serum und im Gewebe, vor allem in der Niere und der Lunge. Dieses Enzym kann in vitro und in vivo RANTES in RANTES(3-68) [21] und Eotaxin in Eotaxin(3-74) [22] umwandeln. Beide Substanzen sind für eosinophile Granulozyten partiell antagonistisch [21-23]. Ein weiteres Beispiel ist die in Hämofiltrat nachgewiesene um 8 Aminosäuren verkürzte Form von HCC-1,
HCC-1(9-74). Diese verkürzte Form kann durch verschiedene, aus Zellkulturüberständen freigesetzte, Peptidasen generiert werden [24]. Die biologische Potenz von HCC-1 ist relativ gering [25], die verkürzte Form, HCC-1(9-74), ist aber chemotaktisch für Lymphozyten, Monozyten und eosinophile Granulozyten [24]. Auf der anderen Seite blockiert HCC-1(9-74) den HIV-Eintritt in Zelllinien, die den CCR5 als Korezeptor exprimieren [24]. Somit können im Gewebe vorkommende Peptidasen, durch Abspaltung von Aminosäuren am N-terminalen Ende der Chemokine, die Rekrutierung der Entzündungszellen durch Generierung antagonistischer oder agonistischer Chemokinderivate steuern.

In eigenen Untersuchungen konnten wir zeigen, dass der Chemokinrezeptor-Antagonist Met-RANTES eine spezifische Inhibition eosinophiler Granulozyten bewirkt. Eine Prästimulation mit Met-RANTES inhibiert die Chemotaxis, Aktinpolymerisation, intrazelluläre $\mathrm{Ca}^{2+}$-Ströme und die Freisetzung reaktiver Sauerstoffspezies nach Stimulation mit RANTES, MCP-3 und Eotaxin [26]. Hierbei blockiert Met-RANTES eosinophile Granulozyten über die Chemokinrezeptoren CCR1 und CCR3 [26]. Darüber hinaus konnte in einem Mausmodell gezeigt werden, dass die Rekrutierung eosinophiler Granulozyten in die Haut durch Injektion von murinem Eotaxin durch Met-RANTES blockiert werden kann [16]. Im Gegensatz zu Met-RANTES hat die chemisch modifizierte Form von RANTES, AOP-RANTES, keine antagonistischen Effekte auf eosinophile Granulozyten. AOP-RANTES aktiviert eosinophile Granulozyten sowohl über den CCR1 als auch über den CCR3. Die Wirkung von AOP-RANTES auf eosinophile Granulozyten ist vergleichbar mit RANTES, aber weniger effektiv als die von Eotaxin oder Eotaxin-2 [27].

In einer neueren Studie konnte ein weiterer CCR3-Antagonist, Met-chemokine beta 7 (Met-Ckbeta7), charakterisiert werden [28]. Dieser Chemokinrezeptor-Antagonist stellt eine modifizierte Form von PARC dar. Met-Ckbeta7 inhibierte in diesen Untersuchungen intrazelluläre $\mathrm{Ca}^{2+}$-Ströme und die Chemotaxis eosinophiler Granulozyten nach Stimulation mit zahlreichen CCR3-Liganden wie Eotaxin [28]. Das synthetisch hergestellte „HybridChemokin“ I-TAC/EoH1 leitet sich vom CC-Chemokin Eotaxin und CXC-Chemokin I-TAC ab [29]. Dieses „Hybrid-Chemokin“ zeigt eine 3fach stärkere Affinität zum Chemokinrezeptor CCR3 als Eotaxin, und es ist ein 5fach stärkerer Antagonist als I-TAC am CCR3. I-TAC/EoH1 hemmte sowohl die Eotaxin-induzierte Chemotaxis als auch die Freisetzung intrazellulären Kalziums ohne eine intrinsische Aktivität aufzuweisen [29]. Diese Untersuchungen zeigen, dass nicht nur die ersten 8 Aminosäuren des $\mathrm{N}$-Terminus von Eotaxin, sondern auch Aminosäuren in der Region nach dem $\mathrm{N}$-Terminus für die Bindung und Aktivierung am CCR3 verantwortlich sind.

Der Vorteil von Peptiden als Chemokinrezeptor-Antagonisten in der Therapie von allergischen Erkrankungen besteht darin, dass sie eine relativ gute Verträglichkeit aufweisen. So werden sie nicht primär in der Leber metabolisiert, und es können somit auch kaum Wechselwirkungen mit anderen Medikamenten auftreten. Da es bereits zahlreiche Beispiele für eine Peptid-Therapie bei anderen Erkrankungen wie Diabetis mellitus (Insulin), Neutropenie (Granulozyten-Kolonien stimulierender Faktor, G-CSF) und renaler Anämie (Erythropoetin) gibt, ist es durchaus realistisch, dass sich modifizierte Chemokine als Inaktivatoren 
oder Antagonisten für allergische Erkrankungen durchsetzen können.

\section{Klein-molekülige Chemokinrezeptor-Antagonisten}

Eine neue/andere Form der Chemokinrezeptor-Antagonisten ist Gegenstand aktueller Forschungsvorhaben zahlreicher Pharmaunternehmen. Hierbei handelt es sich um so genannte kleinmolekülige Chemokinrezeptor-Antagonisten, die keine Peptide darstellen [10,30,31]. Diese Antagonisten besitzen ein kleines Molekulargewicht und beinhalten hydrophobe Gruppen, die sich in der Nähe eines zentralen und für die Funktion verantwortlichen Stickstoffmoleküls befinden. Das zentrale Stickstoffmolekül scheint dabei im oder mit dem 7-transmembranösen Chemokinrezeptor zu interagieren [10].

$\mathrm{Zu}$ diesen klein-moleküligen Chemokinrezeptor-Antagonisten zählt TAK-779, das die Bindung von RANTES an CCR5+-Zelllinien und intrazelluläre $\mathrm{Ca}^{2+}$-Ströme in diesen Zellen blockierte. Darüber hinaus konnte in Studien gezeigt werden, dass TAK-779 die Replikation von HIV-Strängen in Monozyten inhibiert [32,33]. Die Substanzen T134 und AMD3100 wurden als blockierende Antagonisten für den CXC-Chemokinrezeptor CXCR4 beschrieben. Sie blockieren die Bindung eines gegen den CXCR4 gerichteten monoklonalen Antikörpers und verhindern den CXCR4 vermittelten HIV-Eintritt [34]. In weiteren Studien wurden klein-molekülige Chemokinrezeptor-Antagonisten, die gegen den CC-Chemokinrezeptor CCR1 gerichtet waren, untersucht. Die Substanz 1 (compound 1), die auch als CCR1-Antagonist 1 bezeichnet wird, zeigte eine dosisabhängige Inhibition intrazellulärer $\mathrm{Ca}^{2+}-\mathrm{Strö-}$ me nach Stimulation mit MIP- $1 \alpha$ ohne intrinsische Aktivität $[35,36]$.

Ein besonderes Interesse besteht in der Suche nach klein-moleküligen Chemokinrezeptor-Antagonisten gegen den CC-Chemokinrezeptor CCR3. Insbesondere die Firmen Banyu Pharmaceutical, Glaxo-SmithKline, Abbott Laboratories, Bristol-Myers Squibb, Hoffmann-La Roche und Merck haben hier potentielle CCR3-Antagonisten in Aussicht, die sich von den Piperidinen, Piperazinen oder Pyrrolidinen ableiten [10]. Die chemische Modifikation der Komponente SK\&F 45523 führte zur Herstellung der potenten CCR3-Antagonisten SB-297006 und SB-328437 [37]. Diese Antagonisten zeigen eine hohe Affinität zum CCR3 und hemmen die Bindung von $\left({ }^{125}\right)$ I-Eotaxin und $\left({ }^{125}\right)$ I-MCP-4 auf human eosinophilen Granulozyten. Darüber hinaus inhibieren SB-297006 und SB-328437 intrazelluläre $\mathrm{Ca}^{2+}$-Ströme in eosinophilen Granulozyten nach Stimulation mit Eotaxin und MCP-4. Der Antagonist SB-328437 blockiert außerdem die Chemotaxis eosinophiler Granulozyten nach Stimulation verschiedener CCR3-Liganden [37]. Der klein-molekülige ChemokinrezeptorAntagonist UCB35625 zeigt eine selektive inhibierende Wirkung auf die CC-Chemokinrezeptoren CCR1 und CCR3. So inhibiert dieser Antagonist die Formveränderung eosinophiler Granulozyten und die Internalisierung von CCR1 und CCR3 nach Stimulation mit MIP-1 $\alpha$, MCP-4 und Eotaxin [38].

Der Vorteil dieser klein-moleküligen Chemokinrezeptor-Antagonisten besteht vor allem darin, dass sie sich gut für eine orale oder topische Applikation eignen. Schließlich handelt es sich nicht um Peptide, sondern um hydrophobe und damit pharmakologisch gut resorbierbare Substanzen [31]. Der Nachteil dieser
Gruppe von Substanzen besteht aber darin, dass sie in der Leber metabolisiert werden und damit verstärkt Wechselwirkungen mit anderen Medikamenten auftreten können. Darüber hinaus können diese lipophilen Substanzen die Blut-Hirn-Schranke passieren, und damit die von den Antihistaminika bekannten Nebenwirkungen wie Müdigkeit induzieren.

Es ist momentan noch zu früh, um eine Aussage darüber zu machen, ob die klein-moleküligen Chemokinrezeptor-Antagonisten oder modifizierte Chemokine sich therapeutisch besser zur Therapie allergischer Erkrankungen eignen. Aus diesem Grunde besteht weiterer Forschungsbedarf für die Entwicklung von Chemokinrezeptor-Antagonisten oder Chemokinrezeptor-Inaktivatoren. In unserer Arbeitsgruppe beschäftigen wir uns zur Zeit mit der Generierung von modifizierten Chemokinen mit antagonistischer als auch agonistischer Wirkung um eine Inaktivierung von Chemokinrezeptoren zu bewirken. Ein wichtiges Ziel dieser Therapie sollte sein, dass die Einwanderung von Entzündungszellen aus dem Blut frühzeitig gestoppt wird, um die Symptome zu verhindern.

\section{Literatur}

${ }^{1}$ Elsner J, Forssmann U. Another renaissance of eosinophils in allergic diseases. ACI International 2002; 14: 151 - 155

2 Loetscher P, Clark-Lewis I. Agonistic and antagonistic activities of chemokines. J Leukoc Biol 2001; 69: 881 - 884

3 Ponath PD, Qin S, Post TW, Wang J, Wu L, Gerard NP, Newman W, Gerard C, Mackay CR. Molecular cloning and characterization of a human eotaxin receptor expressed selectively on eosinophils. J Exp Med 1996; 183: $2437-2448$

${ }^{4}$ Uguccioni M, Mackay CR, Ochensberger B, Loetscher P, Rhis S, LaRosa GJ, Rao P, Ponath PD, Baggiolini M, Dahinden CA. High expression of the chemokine receptor CCR3 in human blood basophils. Role in activation by eotaxin, mcp-4, and other chemokines. J Clin Invest 1997; 100: $1137-1143$

${ }^{5}$ Ochi H, Hirani WM, Yuan Q, Friend DS, Austen KF, Boyce JA. T helper cell type 2 cytokine-mediated comitogenic responses and CCR3 expression during differentiation of human mast cells in vitro. J Exp Med 1999; 190: 267-280

${ }^{6}$ Sallusto F, Mackay CR, Lanzavecchia A. Selective expression of the eotaxin receptor CCR3 by human T helper 2 cells. Science 1997; 277: $2005-2007$

${ }^{7}$ Humbles AA, Lu B, Friend DS, Okinaga S, Lora J, Al Garawi A, Martin TR, Gerard NP, Gerard C. The murine CCR3 receptor regulates both the role of eosinophils and mast cells in allergen-induced airway inflammation and hyperresponsiveness. Proc Natl Acad Sci USA 2002; 99: $1479-1484$

${ }^{8}$ Ma W, Bryce PJ, Humbles AA, Laouini D, Yalcindag A, Alenius H, Friend DS, Oettgen HC, Gerard C, Geha RS. CCR3 is essential for skin eosinophilia and airway hyperresponsiveness in a murine model of allergic skin inflammation. J Clin Invest 2002; 109: 621 -628

${ }^{9}$ Owen C. Chemokine receptors in airway disease: which receptors to target? Pulm Pharmacol Ther 2001; 14: 193 - 202

${ }^{10}$ Bertrand CP, Ponath PD. CCR3 blockade as a new therapy for asthma. Expert Opin Investig Drugs 2000; 9: 43-52

${ }^{11}$ Gong JH, Clark-Lewis I. Antagonists of monocyte chemoattractant protein 1 identified by modification of functionally critical $\mathrm{NH} 2$-terminall residues. J Exp Med 1995; 181: 631 -640

12 Weber M, Uguccioni M, Baggiolini M, Clark Lewis I, Dahinden CA. Deletion of the NH2-terminal residue converts monocyte chemotactic protein 1 from an activator of basophil mediator release to an eosinophil chemoattractant. J Exp Med 1996; 183: 681-685

${ }^{13}$ Wells TNC, Power CA, Lusti-Narasimhan M, Hoogewerf AJ, Cooke RM, Chung C, Peitsch MC, Proudfoot AEI. Selectivity and antagonism of chemokine receptors. J Leukoc Biol 1996; 59: 53-60

14 Baggiolini M, Moser B. Blocking chemokine receptors. J Exp Med 1997; 186: $1189-1191$ 
${ }^{15}$ Proudfoot AE, Power CA, Hoogewerf AJ, Montjovent MO, Borlat F, Offord RE, Wells TN. Extension of recombinant human RANTES by the retention of the initiating methionine produces a potent antagonist. J Biol Chem 1996; 271: 2599-2603

${ }^{16}$ Teixeira MM, Wells TN, Lukacs NW, Proudfoot AE, Kunkel SL, Williams TJ, Hellewell PG. Chemokine-induced eosinophil recruitment. Evidence of a role for endogenous eotaxin in an in vivo allergy model in mouse skin. J Clin Invest 1997; 100: 1657-1666

17 Simmons G, Clapham PR, Picard L, Offord RE, Rosenkilde MM Schwartz TW, Buser R, Wells TNC, Proudfoot AE. Potent inhibition of HIV-1 infectivity in macrophages and lymphocytes by a novel CCR5 antagonist. Science 1997; 276: 276-279

18 Mack M, Luckow B, Nelson PJ, Cihak J, Simmons G, Clapham PR, Signoret N, Marsh M, Stangassinger M, Borlat F, Wells TNC, Schlondorff D, Proudfoot AEI. Aminooxypentane-RANTES induces CCR5 internalization but inhibits recycling: A novel inhibitory mechanism of HIV infectivity. J Exp Med 1998; 187: 1215 - 1224

${ }^{19}$ Struyf S, De Meester I, Scharpe S, Lenaerts JP, Menten P, Wang JM, Proost P, van Damme J. Natural truncation of RANTES abolishes signaling through the CC chemokine receptors CCR1 and CCR3, impairs its chemotactic potency and generates a CC chemokine inhibitor. Eur J Immunol 1998; 28: $1262-1271$

20 Proost P, Struyf S, Couvreur M, Lenaerts JP, Conings R, Menten P, Verhaert P, Wuyts A, van Damme J. Posttranslational modifications affect the activity of the human monocyte chemotactic proteins MCP- 1 and MCP-2: identification of MCP- 2(6-76) as a natural chemokine inhibitor. J Immunol 1998; 160: 4034-4041

21 Oravecz T, Pall M, Roderiquez G, Gorrell MD, Ditto M, Nguyen NY, Boykins R, Unsworth E, Norcross MA. Regulation of the receptor specificity and function of the chemokine RANTES (regulated on activation, normal $\mathrm{T}$ cell expressed and secreted) by dipeptidyl peptidase IV (CD26)- mediated cleavage. J Exp Med 1997; 186: 1865-1872

22 Struyf S, Proost P, Schols D, De Clercq E, Opdenakker G, Lenaerts JP, Detheux M, Parmentier M, De M, Scharpe IS, Van Damme J. CD26/dipeptidyl-peptidase IV down-regulates the eosinophil chemotactic potency, but not the anti-HIV activity of human eotaxin by affecting its interaction with CC chemokine receptor 3. J Immunol 1999; 162: $4903-4909$

23 Proost P, De Meester I, Schols D, Struyf S, Lambeir AM, Wuyts A, Opdenakker G, DeClercq E, Scharpe S, Van Damme J. Amino-terminal truncation of chemokines by CD26/dipeptidyl-peptidase IV. Conversion of RANTES into a potent inhibitor of monocyte chemotaxis and HIV-1-infection. J Biol Chem 1998; 273: 7222 - 7227

24 Detheux M, Standker L, Vakili J, Munch J, Forssmann U, Adermann K, Pohlmann S, Vassart G, Kirchhoff F, Parmentier M, Forssmann WG. Natural proteolytic processing of hemofiltrate CC chemokine 1 generates a potent CC chemokine receptor (CCR) 1 and CCR5 agonist with antiHIV properties. J Exp Med 2000; 192: 1501 - 1508

${ }^{25}$ Schulz-Knappe P, Magert HJ, Dewald B, Meyer M, Cetin Y, Kubbies M, Tomeczkowski J, Kirchhoff K, Raida M, Adermann K, Kist A, Reinecke M, Sillard R, Pardigol A, Uguccioni M, Baggiolini M, Forssmann WG. HCC-1, a novel chemokine from human plasma. J Exp Med 1996; 183: 295-299

${ }^{26}$ Elsner J, Petering H, Hochstetter R, Kimmig D, Wells TN, Kapp A, Proudfoot AE. The CC chemokine antagonist Met-RANTES inhibits eosino- phil effector functions through the chemokine receptors CCR1 and CCR3. Eur J Immunol 1997; 27: $2892-2898$

${ }^{27}$ Elsner J, Mack M, Bruhl H, Dulkys Y, Kimmig D, Simmons G, Clapham PR, Schlondorff D, Kapp A, Wells TN, Proudfoot AE. Differential activation of CC chemokine receptors by AOP-RANTES. J Biol Chem 2000; 275: $7787-7794$

${ }^{28}$ Nibbs RJ, Salcedo TW, Campbell JD, Yao XT, Li Y, Nardelli B, Olsen HS, Morris TS, Proudfoot AE, Patel VP, Graham GJ. C-C chemokine receptor 3 antagonism by the beta-chemokine macrophage inflammatory protein 4 , a property strongly enhanced by an amino-terminal alaninemethionine swap. J Immunol 2000; 164: 1488-1497

${ }^{29}$ Loetscher P, Pellegrino A, Gong JH, Mattioli I, Loetscher M, Bardi G, Baggiolini M, Clark-Lewis I. The ligands of CXC chemokine receptor 3, I-TAC, Mig, and IP10, are natural antagonists for CCR3. J Biol Chem 2001; 276: $2986-2991$

${ }^{30}$ Horuk R, Ng HP. Chemokine receptor antagonists. Med Res Rev 2000; 20: $155-168$

${ }^{31}$ Schwarz MK, Wells TN. New therapeutics that modulate chemokine networks. Nat Rev Drug Discov 2002; 1: 347-358

32 Baba M, Nishimura O, Kanzaki N, Okamoto M, Sawada H, Iizawa Y, Shiraishi M, Aramaki Y, Okonogi K, Ogawa Y, Meguro K, Fujino M. A smallmolecule, nonpeptide CCR5 antagonist with highly potent and selective anti-HIV-1 activity. Proc Natl Acad Sci USA 1999; 96: 5698 - 5703

${ }^{33}$ Shiraishi M, Aramaki Y, Seto M, Imoto H, Nishikawa Y, Kanzaki N, Okamoto M, Sawada H, Nishimura O, Baba M, Fujino M. Discovery of novel, potent, and selective small-molecule CCR5 antagonists as antiHIV-1 agents: synthesis and biological evaluation of anilide derivatives with a quaternary ammonium moiety. J Med Chem 2000; 43: 2049-2063

${ }^{34}$ Arakaki R, Tamamura H, Premanathan M, Kanbara K, Ramanan S, Mochizuki K, Baba M, Fujii N, Nakashima H. T134, a small-molecule CXCR4 inhibitor, has no cross-drug resistance with AMD3100, a CXCR4 antagonist with a different structure. J Virol 1999; 73 : $1719-1723$

${ }^{35}$ Hesselgesser J, Ng HP, Liang M, Zheng W, May K, Bauman JG, Monahan S, Islam I, Wei GP, Ghannam A, Taub DD, Rosser M, Snider RM, Morrissey MM, Perez HD, Horuk R. Identification and characterization of small molecule functional antagonists of the CCR1 chemokine receptor. J Biol Chem 1998; 273: 15687-15692

${ }^{36}$ Liang M, Rosser M, Ng HP, May K, Bauman JG, Islam I, Ghannam A, Kretschmer PJ, Pu H, Dunning L, Snider RM, Morrissey MM, Hesselgesser J, Perez HD, Horuk R. Species selectivity of a small molecule antagonist for the CCR1 chemokine receptor. Eur J Pharmacol. 2000; 389: $41-49$

${ }^{37}$ White JR, Lee JM, Dede K, Imburgia CS, Jurewicz AJ, Chan G, Fornwald JA, Dhanak D. Christmann LT, Darcy MG, Widdowson KL, Foley JJ, Schmidt DB, Sarau HM. Identification of potent, selective non-peptide CC chemokine receptor-3 antagonist that inhibits eotaxin-, eotaxin-2-, and monocyte chemotactic protein-4-induced eosinophil migration. J Biol Chem 2000; 275: 36626-36631

38 Sabroe I, Peck MJ, Van Keulen BJ, Jorritsma A, Simmons G, Clapham PR, Williams TJ, Pease JE. A small molecule antagonist of chemokine receptors CCR1 and CCR3. Potent inhibition of eosinophil function and CCR3-mediated HIV-1 entry. J Biol Chem 2000; 275: 25985 - 25992 